Voix et Images

volxetimages

\title{
Une belle journée d'avance de Robert Lalonde ou Quand le roman se fait poésie
}

\section{Madeleine Frédéric}

Volume 15, numéro 1 (43), automne 1989

Jacques Poulin

URI : https://id.erudit.org/iderudit/200818ar

DOI : https://doi.org/10.7202/200818ar

Aller au sommaire du numéro

Éditeur(s)

Université du Québec à Montréal

ISSN

0318-9201 (imprimé)

1705-933X (numérique)

Découvrir la revue

Citer cet article

Frédéric, M. (1989). Une belle journée d'avance de Robert Lalonde ou Quand le roman se fait poésie. Voix et Images, 15(1), 83-92.

https://doi.org/10.7202/200818ar d'utilisation que vous pouvez consulter en ligne.

https://apropos.erudit.org/fr/usagers/politique-dutilisation/ 


\title{
Une belle journée d'avance de Robert Lalonde ou Quand le roman se fait poésie
}

\author{
par Madeleine Frédéric, Université libre de Bruxelles
}

Très beau roman de Robert Lalonde, Une belle journée d'avance ${ }^{1}$ se présente sous la forme d'un récit fait par un je narrateur qui est en même temps un personnage de l'histoire (et même le personnage principal). Le récit de ce narrateur correspond à une plongée dans son passé qui se voit réactualisé dans la narration, notamment par le recours quasi généralisé au présent. On voit dès lors coexister dans le texte un présent véritable, lorsque la narration et l'histoire coïncident: Je reviens au livre, les mots embués tremblent devant moi. $J$ 'ai laissé la fenêtre ouverte, je veux continuer le livre avec la musique de vos rires, en bas (p. 52), et un présent faux ou pseudo-présent, lorsque l'histoire précède la narration, mais que celle-ci, dans la mesure où elle est faite au présent, semble donner comme actuels des actions ou des faits passés. C'est sur une telle fausse piste que s'ouvre précisément le roman:

Je ne suis pas encore né. Pas même dans l' ouf encore. Pas encore ce petit têtard grouillant. Pas encore des vôtres. Cependant, c'est pour très bientôt. Je fais, comme on dira chez nous, "Pâques avant les Rameaux». Je célèbre tout de suite, hanté par elle, au fond de mes limbes, la vie qui vient. (p. 11)

En réalité, trente-sept ans séparent le moment où le narrateur énonce ces lignes de l'époque qu'elles évoquent.

L'effet d'une telle technique est d'opérer une véritable mise à plat du temps, une confusion des différentes époques, qui peut même entraîner une certaine ambiguîté, comme dans la dernière séquence du chapitre intitulé «L'après-midi». Les premières phrases du paragraphe final, Mon amour, la chambre claire est prête. Elle m'attend. Elle attend l'enfant (p. 125), permettent en effet deux lectures: soit le pronom me et le syntagme l'enfant renvoient tous deux anaphoriquement au je narrateur ${ }^{2}$, interprétation que rend possible le paragraphe qui précède et notamment des phrases comme: À l'heure qu'il est, moi, je suis encore dans les limbes. [...] Petit auf vide encore, lové dans le ventre de

1 Robert Lalonde, Une belle journée d'avance, Paris, Seuil, 1985.

2 Pour les notions d'anaphore et de coréférence, lire notamment Madeleine Frédéric, la Répétition. Étude linguistique et rhétorique, Tubingen, Max Niemeyer, 1985, p. 86 et suivantes. 
Gertrude, $j$ 'attends. J' attends l' amour de mon père, son éclair, la fécondation comme on dit. (p. 124) Le me et l'enfant désigneraient alors l'enfant qui naîtra (qui est né et devenu adulte au moment de la narration) des amours de Maurice et Gertrude. Mais elle autorise tout aussi bien une lecture dans laquelle me et l'enfant n'ont pas la même référence, le syntagme renvoyant cette fois à l'enfant à naître des amours du je narrateur et de sa compagne, qu'il désigne par le pronom tu (enfant virtuel sur le plan aussi bien de la diégèse que de la narration). Cette interprétation est suggérée par le contexte immédiat du paragraphe: non seulement par les multiples références explicites au tu (l'apostrophe initiale Mon amour, l'impératif Viens et l'affirmation [C'est] le moment que tu préfères); mais surtout par l'annonce de la remise à plus tard de l'écriture, sur laquelle s'achèvent la séquence et le chapitre.

Il ne faut pas voir ici une simple gymnastique de linguiste en mal d'exercices. Bien au contraire, il me semble important d'épingler ce passage dans l'optique qui nous retient: celle de la confusion des époques. La première lecture correspond, en effet, à un présent diégétique passé en réalité au moment de la narration; tandis que la seconde fait intervenir un présent diégétique tout à fait contemporain de cette narration. Mais ce n'est pas tout, car la confusion déborde en fait le cadre temporel pour s'étendre aux personnages eux-mêmes: le je narrateur et son enfant à venir sont - on vient de le voir - difficiles à distinguer sur le strict plan de la coréférence linguistique. On touche du même coup à un aspect essentiel de l'œuvre, sur lequel on aura l'occasion de revenir plus en détail, l'idée de la continuation des personnages: Gertrude et Maurice se perpétuent dans le je narrateur $(\simeq$ l'enfant 1 ) qui se perpétuera lui-même grâce au tu, dans l'enfant à venir ( $\simeq$ l'enfant ${ }_{2}$ ).

À ce titre, on se trouverait donc devant un passage clé de l'œuvre, un passage «marqué» en quelque sorte par rapport au reste de l'œuvre, dans la mesure où, généralement, chaque séquence correspond à un ou des personnages et à un moment bien précis. Parfois, cependant, on assiste à une convocation «anachronique» de personnages en une même séquence; mais, dans ce cas, le lecteur dispose d'indices lui permettant de s'y retrouver.

C'est le cas, notamment, quelques pages auparavant (p. 119-122). La séquence réunit la tante Malvina, le je, le tu et l'enfant à venir et, du même coup, le passé, le présent et le futur par rapport au moment de la narration.

Cependant, le décalage temporel est relativement clair, dès lors que le narrateur utilise le présent en relation avec le moment présent et le couple je-tu (on se trouve donc en présence d'un véritable présent: le présent de la diégèse est en même temps celui de la narration). Quant à l'usage du futur, il est réservé à la mention qui est faite de l'enfant à naître, tout à la fin de la séquence. Enfin, lorsque le narrateur fait référence au passé et à sa tante Malvina, il utilise principalement l'imparfait. On rencontre également ici et là le plus-que-parfait, une occurrence du passé simple et un présent historique. Ou, ce qui est plus intéressant encore, l'usage du passé composé qui permet un rapprochement avec le présent; mais pas une adéquation, en tout cas pas une assimilation comme celle, fallacieuse, qu'amorce ailleurs le «pseudo-présent». 
D'autre part, si l'on examine la répartition temporelle dans la séquence, on constate que la référence au passé occupe les trois premiers paragraphes, celle qui est faite au présent domine les deux suivants; quant à la brève référence au futur, elle se limite - on l'a vu - à la dernière phrase de la séquence. Force nous est donc de constater que cette répartition reflète fidèlement la succession chronologique.

Dans le chapitre suivant, une séquence (p. 140-142) opère un rapprochement explicite entre deux circonstances semblables: la trace de sang laissée par le maringouin sur le bras du je narrateur et celle du chevreuil écrasé, plusieurs années auparavant, par le camion de ses parents. Mais ici encore le lecteur dispose d'un faisceau d'indices lui permettant de restituer la véritable chronologie: répartition temporelle (présent d'une part, imparfait et passé composé de l'autre), choix des préfixes et appoint lexical: vif comme l'éclair, resurgit l'autre sang et plus loin le vieux signe tuméfié est réapparu, convergent en ce sens.

En revanche, dans la séquence qui nous préoccupe, ce n'est pas le cas. $\mathrm{La}$ coexistence du présent vrai et du «pseudo-présent», ainsi que l'ambiguité coréférentielle soigneusement ménagée autour du syntagme l'enfant, contribuent conjointement à semer le doute chez le lecteur.

Il est important d'ailleurs de remarquer que cette tendance à fondre des époques et des personnages différents se trouve comme amplifiée par la structure même du roman, qui déplie les divers moments d'une journée dans la vie de ces personnages. Ces derniers sont saisis à des moments successifs, consignés ici et là dans la narration et désignés explicitement par le titre des chapitres. Au niveau de la diégèse, il y a donc progression strictement parallèle des personnages; mais celle-ci gagne également le plan de la narration, dès lors que la journée du couple je-tu se révèle elle aussi scandée de la même manière (à trente-sept ans d'intervalle, rappelons-le), comme le montrent notamment les extraits suivants:

Dans le chapitre «L'avant-midi»: Ce matin [...] c'est toi, mon amour, qui chantes (p. 63), et plus encore, dans les dernières pages, le glissement qui s'opère de la sieste - passée - des deux cousins Germain et Jacob: Il est midi, la grosse cloche de l'église sonne l'angélus. (p. 73) Il est midi et le soleil s'amuse de tant d'innocence, de ce péché de tendresse qui commence entre les deux cousins (p. 74), à la situation présente du je narrateur: Oui, il est midi [...]. Je descends te rejoindre sur la grève ( $\mathrm{p}$. 74), par le biais d'une stricte reprise lexicale qui souligne d'autant l'assimilation des deux plans.

Dans le chapitre «L'après-midi», une première séquence consacrée au couple je-tu s'ouvre par une reprise du titre: Cet après-midi [...], je t'ai parlé du marchand général. (p. 109) On reviendra sous peu à cet épisode du marchand général, qui a son importance dans l'optique suivie ici. Une nouvelle référence au titre sera faite à l'extrême fin du chapitre, en relation explicite avec le tu: C'est la fin de l'après-midi, le moment que tu préfères. (p. 125) Or, on se trouve ici dans le tout dernier paragraphe, celui précisément qui renferme les 
phrases ambiguës: Elle $m^{\prime}$ attend. Elle attend l'enfant, examinées plus haut. Le passage est donc à nouveau marqué par rapport au reste. Impression que vient accentuer le fait que ce même dernier paragraphe renferme un nouveau facteur d'assimilation passé-présent: la mention de l'orage qui a touché, dans ce même chapitre, tous les personnages de l'histoire passée et que l'on retrouve en relation avec le couple je-tu et l'époque présente cette fois-ci: La fenêtre est grande ouverte sur un ciel lavé par l'orage. (p. 125)

Dans le chapitre «La brunante», l'unité de temps touche également le je et le tu: Je l'aide à remonter dans le canoë. Le soir tombe, nous avironnons vers la maison blanche. (p. 131)

Enfin, dans le chapitre «La nuit», une séquence consacrée au couple je-tu s'ouvre par la phrase: Autre nuit, même nuit, la nôtre (p. 170), qui met explicitement en relation leur nuit avec celle de Germain et Jacob qui vient d'être évoquée. On s'en souviendra, ce n'est pas la première fois que le narrateur établit un parallèle entre ces deux couples de personnages: dans le chapitre «L'avant-midi» déjà, c'est la reprise (Il est midi... Oui, il est midi) qui permettait le glissement d'un plan (celui des cousins Germain et Jacob, et de l'épisode - passé par rapport au moment de la narration - de leur mort) à l'autre (celui du couple je-tu, et d'un épisode contemporain de la narration).

La confusion entre les personnages et les époques est encore accentuée par tout un jeu de symétries et de rappels entre certaines séquences, le plus net étant probablement celui qui rapproche la séquence initiale du premier chapitre: Tu me dis: «Reste encore mon amour.» Alors je te redonne mon grand poids doux, je m'abandonne sur tes seins, sur ton ventre mouillé de nos sueurs, je souffle fort comme la vague contre ton oreille (p. 15), et la séquence finale du dernier: Gertrude dit: «Reste encore, mon amour.» Alors il lui redonne son grand poids doux, il s'abandonne sur ses seins, sur son ventre mouillé de leurs sueurs, il souffle fort, comme la vague, contre son oreille. (p. 182)

La première correspond à une narration simultanée (le présent de la narration et le présent de l'histoire coïncident), alors que la seconde fait apparaître une narration ultérieure (le présent de la narration correspond au passé de l'histoire); pourtant, l'une et l'autre sont au présent. On retrouve dès lors la technique, précédemment évoquée, de mise à plat, d'égalisation temporelle.

En outre, le jeu des reprises lexicales contribue à assimiler ces deux passages: on passe des deux premières phrases de la séquence initiale à leurs homologues dans la demière, par simple substitution de Gertrude à tu et de il (= Maurice) à je. On assiste dès lors à une identification entre les deux couples; identification implicite mise en valeur par la distribution des séquences dans le texte puisque, rappelons-le, l'une ouvre le premier chapitre, l'autre ferme le dernier.

Cette identification ne restera d'ailleurs pas qu'à un niveau implicite: deux épisodes contribueront à l'imposer, plus exactement à la projeter du plan de la narration à celui de l'histoire. Et - faut-il voir là une simple coïncidence? ces deux épisodes sont concentrés dans le chapitre «L'après-midi», dont on a vu 
à suffisance qu'il s'achevait sur un paragraphe hautement significatif dans l'économie générale du roman.

Dans le premier épisode, la compagne du narrateur déniche au grenier une robe qui se révèle être la robe de maternité de Gertrude. Elle s'empresse de l'essayer en présence du narrateur qui écrit:

Je te regarde, je ne dis rien. Les desseins du grand hasard me font tressaillir. Ce n'est pas tellement la robe, mon amour. C'est que tu lui ressembles beaucoup et que, soudain, le temps est aboli, la mémoire inutile, inutiles les vieilles photos. Pire encore: inutiles mes efforts pour recréer Gertrude, dans le livre. (p. 93)

Le second épisode est celui du marchand général qui, en présence du narrateur et de sa compagne, croit revoir en eux les parents du narrateur (p. 109). Celui-ci conclura son récit par cette affirmation laconique: Pour ce qui est de la ressemblance, il a raison, bien sûr, le marchand général. Mais ça, nous le savions déjà. On rejoint ici une hypothèse déjà avancée à propos du dernier paragraphe de ce même chapitre: l'idée d'une continuation des personnages ${ }^{3}$. Ce thème sera déplié à diverses reprises dans le roman: Imagine une goutte de rosée [...] qui ne sait pas où elle aboutira [...], et même si elle ne s'évaporera pas: c'est moi, mon amour. Et c'est peut-être déjà l' enfant en toi, aussi. (p. 35) L'enfant, ce sera eux [les parents du narrateur] encore, eux recommencés. (p. 179)

Ce tout dernier extrait est emprunté à ce qui est sans nul doute l'épisode capital de l'œuvre: celui dans lequel le narrateur accepte d'évoquer pour sa compagne le suicide de ses parents, survenu alors qu'il avait quinze ans à peine. Capitale, cette séquence l'est parce qu'elle convoque les personnages essentiels pour le narrateur: ses parents, la compagne salvatrice et leur futur enfant. Elle réunit du même coup plusieurs époques: la vie passée du narrateur, le redémarrage de sa vie correspondant à l'arrivée de sa compagne, et sa vie future qui verra se perpétuer leur couple dans l'enfant à naître. Capital, l'épisode l'est peut-être plus encore parce que ce suicide a entrainé chez le narrateur un refoulement complet, un silence qui durera vingt ans et qui est rompu ici pour la première fois. La séquence s'achève d'ailleurs sur ces mots: C'est fini maintenant. L'enfant est commencé, le monde est de nouveau le monde, le nouveau monde. L'été s'est allumé sur ton corps, sur le mien. C'est fini maintenant. Il fallait simplement le dire, l'écrire. C'est fini. (p. 179-180) On assiste dès lors à l'affirmation d'une triple catharsis: par la parole, par l'écriture et par la paternité.

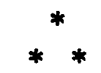

3 Cf. supra: perpétuation de Gertrude et Maurice dans le narrateur, qui se perpétuera lui-même dans l'enfant à venir. 
L'approche essentiellement narratologique qui précède pourrait laisser croire que le ton de l'œuvre est clair, que son statut de roman est évident. Et pourtant, il n'en est rien, car certains passages semblent montrer des affinités très nettes avec la poésie.

Cette dernière est à entendre, ainsi que le font Joëlle Tamine et Jean Molino, comme: [...] l'application d'une organisation métrico-rythmique sur l'organisation linguistique. Pour ces auteurs,

la poésie nât de l'application du rythme sur le langage [...]; elle est le langage plus autre chose qui n'est pas spécifiquement linguistique, et qui est le rythme, le rythme que l'on trouve dans le mouvement, la danse ou la musique, et qui, s'appliquant sur le langage, le soumet da une élaboration qui a le statut d'une véritable mutation. La poésie n'est pas, elle est le résultat d' une construction. ${ }^{4}$

C'est bien le cas en différents passages de l'œuvre et, tout particulièrement, dans ce qu'il conviendrait d'appeler le discours embryonnaire. J'entends par là l'ensemble des séquences dans lesquelles le je narrateur prend la parole en tant qu'embryon. Toutes ces séquences présentent, en effet, une construction qui, par sa densité même, tend à détourner le regard d'une lecture linéaire vers une lecture tabulaire.

Elles font intervenir des modalités extrêmement diverses et parfois complexes de répétition: reprises phoniques, répétitions lexicales en tout genre, répétitions syntaxiques pouvant aller jusqu'au couplage, reprises syllabiques ou encore répétitions sémantiques ${ }^{5}$ parcourent ces différentes séquences en les structurant.

Il ne peut être question d'entrer ici dans le détail de l'analyse. J'aimerais toutefois épingler deux passages qui me semblent particulièrement intéressants à cet égard, dont la séquence embryonnaire qui figure dans le chapitre «L'avantmidi» (p. 34-35), où le lecteur est véritablement entraîné de maille en maille jusqu'à la fin. Cette séquence s'écoule inéluctablement, en même temps qu'un effet d'accélération y est assuré efficacement par l'enchaînement de reprises de toutes espèces. À titre d'exemple, retenons la succession de propositions tétrasyllabiques qui constituent la cinquième phrase: Je n'attends pas; je suis rythme, je suis liquide, je suis fuyant, je suis rapide, je suis en chemin. (p. 34) La répétition syllabique est elle-même appuyée par la reprise lexicale: d'abord limitée à la seule anaphore de je dans la première proposition, elle s'étend à tout le syntagme verbal (je suis) dans les suivantes, qui déterminent en outre un couplage strict (permanence du moule syntaxique: sujet-verbe-attribut). Il y a,

4 Jean Molino et Joëlle Tamine, Introduction à l'analyse linguistique de la poésie, Paris, P.U.F., 1982, p. 8-9.

5 Pour une typologie plus complète de la répétition, lire Madeleine Frédéric, op. cit., chapitre 4; pour la notion de couplage, lire entre autres, Madeleine Frédéric, la Répétition et ses structures dans l'cuvre poétique de Saint-John Perse, Paris, Gallimard, 1984. 
d'autre part, échange de significations entre tous ces adjectifs ou termes adjectivés, qui concourent à véhiculer cette même idée d'écoulement rapide et inexorable: on retrouve au niveau local de la phrase le contenu et les techniques qui caractérisent toute la séquence. Le mouvement se prolonge d'ailleurs dans la phrase suivante, qui est bâtie sur une triade épithétique - chaque adjectif comportant trois syllabes et les deux premiers étant proches par les sonorités (allitération en [1] et assonance en [y]): Lumineux, fulgurant, nécessaire. Elle marque le retour de la construction nominale, que l'on rencontrait dans les phrases 2 et 3 et qui contribue encore au mouvement d'accélération. Celui-ci ne fera que s'accentuer par la suite, en raison de l'enchaînement des énumérations, relayées ici et là par la figure de l'accumulation. Il culminera en outre avec le raccourci temporel fulgurant (déjà signalé) sur lequel s'achève la séquence: Imagine une goutte de rosée [...]; c'est moi, mon amour. Et c'est peut-être déjà l'enfant en toi, aussi.

Le deuxième «passage» qui nous retiendra regroupe en fait trois séquences embryonnaires qui doivent, à mon avis, être examinées en bloc, dès lors qu'elles sont rapprochées: par leur distribution en un même chapitre, le demier, intitulé «La nuits, par la permanence de certains thèmes, mais surtout par leur parenté formelle, assurée de manière efficace par l'anaphore je suis et sa variante je serai.

La première de ces séquences (p. 153-154) est nettement placée sous le signe de la dualité. La construction binaire préside en effet à un grand nombre de phrases: il suffit d'ailleurs de lire les premières lignes pour s'en convaincre:

Je serai peut-être homme et femme? Je suis déjd mâle et femelle et plus encore, aquatique et filant vite, indéfini, ambigu, à cause du temps qui n' existe pas encore. Choisir est impossible. Je coule, je me faufile dans la tendresse brûlante de l'eau de mère. Je suis tour à tour dérobé et enrobé, petit mâle, petite femelle.

Le mouvement binaire y est assuré par le jeu de paires antithétiques, telles homme et femme, mâle et femelle, dérobé et enrobé, petit mâle, petite femelle, et traduit également par le choix d'un terme comme ambigu, qui sera d'ailleurs repris et amplifié quelques lignes plus loin dans la triade: amphibie, ambivalent, ambigu (qui voit le retour du même préfixe).

Ces facteurs de structuration manifestes sont, ici encore, sous-tendus par un réseau phonique qui, à un niveau plus discret mais non moins efficace, «distille» une même cohérence:

aquatique et filant vite: l'assonance en [i], partiellement doublée par l'allitération en [t], renforce la cohésion du groupe, qu'assurait déjà la présence du jonctif et;

elle sera tantôt fluide et tantôt figée, claire et brouillée comme le ruisseau avant et après un gros coup de vent de printemps: les allitérations en [f], [1] et [r] se voient appuyées par les rimes (figée - brouillée et avant - vent - printemps);

rosée, filtrée, mais rutilante: allitérations (en [r] et partiellement en [t] et [1]) et rime (rosée - filtrée) convergent dans cette triade épithétique que vient en outre sous-tendre la répétition syllabique $(2+2+4$ syllabes); 
Fusion, feux doux, appel: cette triade substantive, dont les deux premiers constituants sont unis par l'allitération en [f], fait apparaître une répétition syllabique stricte (succession de bisyllabes).

La cohérence interne de la deuxième séquence (p. 161-162) repose essentiellement sur l'anaphore Je suis, dont les occurrences sont à ce point abondantes qu'elles semblent rapprocher ce passage des litanies. Celui-ci fait insensiblement penser au Cantique des cantiques, non seulement en raison de l'anaphore Je suis, mais également par le jeu des comparaisons et la prédominance du thème de l'amour. On notera le glissement de plan qui s'opère ici des amants au fruit de leur amour; le thème de l'amour reçoit de ce fait même un éclairage neuf, dès lors qu'il ne s'agit plus d'un hommage de l'amant à l'aimée, mais de l'embryon qui se définit uniquement par référence aux deux amants. Le lien avec la tradition est donc maintenu, mais déplacé.

Dans la troisième séquence (p. 180-182), l'anaphore je suis réapparaît; mais elle est nettement moins représentée que dans la séquence précédente et, la plupart du temps, elle se réduit à la simple reprise du pronom je. La structuration serait ici plutôt du même type que dans la toute première séquence examinée (p. 34-35): le lecteur est entraîné de maille en maille jusqu'à la fin. Sans entrer dans le détail de l'analyse, ce qui nous mènerait trop loin et risquerait de ne déboucher que sur un inventaire mécanique et ennuyeux, bornons-nous à constater que répétition, énumération et accumulation convergent une nouvelle fois pour assurer au texte un écoulement continu et inexorable.

Il est intéressant d'observer que ce dernier passage trouve un prolongement, un écho dans la brève séquence qui suit et sur laquelle s'achève significativement le demier chapitre. Au premier regard, la séquence semble d'abord consacrée aux parents du je uniquement. Mais celui-ci se voit bientôt convoqué à son tour et tous trois sont fondus en un nous réconciliateur: Les étoiles sont à nous trois, la brise est a nous trois. (p. 182) La «solidarité» des deux séquences est encore renforcée par la permanence du thème de la mer: le champ lexical, mer, algue, vague, coraux, sable, coquillages, etc., déplié dans la séquence précédente, se perpétue ici au travers des termes vague, grève et houle.

Cette dernière observation nous amène à l'examen d'une autre caractéristique de l'œuvre. En effet, si, comme on vient de le voir, la structuration interne de chaque séquence embryonnaire est manifeste (et «manifestée», pourrait-on dire), on rencontre également des jalons de séquence à séquence. Signalons, à titre d'exemple:

Imagine un souffle qui cherche une bouche, une étincelle qui court dans le champ, un petit espoir très féroce: c'est moi! (p. 11)

Imagine une goutte de rosée pleine du désir d'étancher les soifs multiples des végétaux et qui fuit dans le vent, qui se perd dans un ciel furieux, qui vole dans la tempête, qui ne sait pas où elle aboutira, dans quelle corolle, quelle foliole, et même si elle ne s'évaporera pas: c'est moi, mon amour. Et c'est peut-être déjà l'enfant en toi, aussi. (p. 34-35) 


\section{ÉTUDES 91}

Ce ne sont pas seulement les diverses reprises lexicales (Imagine, qui, c'est moi) et le recours à un même type de construction qui rapprochent ces deux phrases, mais la distribution de l'une et l'autre à la fin de chaque séquence précisément, donc à une place nettement privilégiée. Signalons que l'anaphore Imagine sera reprise, mais seule cette fois, dans la dernière phrase du dernier chapitre (donc dans une distribution semblable à celle des deux occurrences précédentes): Imagine comme je saurai l' accueillir; je connais enfin la douleur et le plaisir, moi aussi, la vie. (p. 182) Retenons encore les jalons suivants: [Je ne suis] pas encore ce petit têtard grouillant (p. 11); Oui, je serai d'abord ce petit têtard fragile [...] (p. 125); Je suis un petit têtard pétrifié, bouleversé de spasmes mais immobile (p. 181); et Pas même dans l' euf encore (p. 11); Petit cuuf vide encore, lové dans le ventre de Gertrude, j'attends (p. 124).

J'aimerais attirer encore l'attention sur des similitudes significatives existant entre les deux séquences embryonnaires qui se relaient à la fin du dernier chapitre ${ }^{6}$ et l'ultime page de l'œuvre. Une petite tache qui danse dans ta respiration immense (p. 183), renvoie symétriquement à: Dans sa respiration immense, une petite tache qui danse. (p. 180) N'était le passage de ta à sa, la correspondance serait parfaite. De même, Aveugle, il voit par ta peau, par ton sang, il nous voit (p. 183) reprend en raccourci: Aveugle, je vois par le sang, je vois par la peau diaphane de mon monde, je vois comme je verrai sous l'eau, avec mes yeux salés. (p. 180) D'autres symétries sont plus discrètes:

La nuit palpite doucement au-dessus de nous. Les étoiles sont à nous trois, la brise est à nous trois. Tout $m^{\prime}$ attend maintenant. Je vais grandir, pousser, venir rejoindre les autres, durer mon temps, remplir mon espace, compter. (p. 182)

Comme un plongeur qui a touché le fond avec sa tête, ébloui, presque suffoqué, secoué d'un long frisson de triomphe qui m'intimide beaucoup, je te rejoins dans la clarté et je me tais. Maintenant, c'est lui qui compte. (p. 183)

La distribution identique en fin de paragraphe, la parenté sémantique unissant les autres termes nuits - étoiles - clarté, et le retour du verbe compter comme demier mot du paragraphe (qui est aussi, dans le second extrait, le dernier mot du livre), tout ceci contribue à attirer l'attention sur cette nouvelle triade: je - tu - il, symétrique de la précédente: Maurice - Gertrude - je. Le je n'est plus l'enfant à naître, mais parent en puissance; quant à la troisième personne, elle ne réfère plus cette fois aux parents du narrateur, mais à l'enfant qui naîtra de lui.

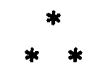

6 Cf. p. $180-181$ et p. 182 évoquées précédemment. On peut voir dans ce dernier élément un nouveau facteur de rapprochement entre les deux séquences, au même titre que la permanence du thème de la mer. 
En définitive, ces multiples jeux de reprises et de symétries nous ramènent, après un long détour, au thème de la continuation des personnages. On voit ainsi peu à peu s'assembler les pièces de ce superbe puzzle. On l'a constaté, en effet, la narration tout entière correspond à une plongée dans le passé; cette plongée, refusée pendant vingt ans par le narrateur, va lui permettre en le nommant, d'évacuer le drame de la mort de ses parents. Mais cette révélation, qui est en même temps une libération, n'intervient que fort tard: ce n'est que dans le tout dernier chapitre que le narrateur accepte de parler. Dans cet épisode capital où il relate à sa compagne le suicide de ses parents, le narrateur se réconcilie - pourrait-on dire - avec sa vie d'enfant; plus exactement, il fait retrouver à cette dernière sa cohérence profonde. Après avoir gommé tout ce passé pendant vingt ans, il en réassemble les pans; il reprend le fil de son histoire et de sa vie à l'époque et à l'endroit précis où il l'avait laissé (on se rappellera, en effet, que son silence s'est accompagné d'une fuite loin du village natal). Ici, dès lors qu'il renoue, physiquement et mentalement, avec cette enfance, le nivellement temporel, la convocation anaphorique des personnages, ainsi que les jeux de symétries et de rappels trouvent du même coup leur raison d'être.

La révélation finale explique peut-être aussi en retour le statut privilégié des séquences embryonnaires, qui se détachent des autres par leur «ton poétique»; dans ces séquences, c'est moins ce qui se passe qui est important que les jeux de rythme et les notations d'impressions de toutes sortes. Or, cette situation embryonnaire correspond très nettement pour le narrateur au moment de l'innocence, du bonheur qui ont précédé la catastrophe. À ce stade, le narrateur est encore intégré à la triade amoureuse familiale, il partage encore le bonheur du couple, ce qui lui sera définitivement refusé par le suicide de ses parents, qui l'excluent du même coup brutalement de ce microcosme bienheureux. On comprend dès lors qu'il accorde à l'évocation de tels moments une place de choix.

C'est la venue de sa compagne qui va progressivement le réconcilier avec la vie, lui permettre de nommer et d'évacuer la cassure brutale survenue dans son enfance, et enfin de retrouver, de reconstituer à son tour une nouvelle triade amoureuse.

Assez significativement, c'est sur l'évocation de la situation embryonnaire - image même de la sérénité totale - que s'ouvre et se ferme tout le roman. Du bonheur passé du narrateur à celui, futur, qu'il ménage pour son enfant, l'œuvre prouve ainsi toute sa cohérence. 